\title{
sciendo
}

\section{Influence of Inspiratory Muscle Training of Various Intensities on The Physical Performance of Long-Distance Runners}

\author{
by \\ Krystyna Rożek-Piechura ${ }^{1}$, Monika Kurzaj ${ }^{1}$, Paulina Okrzymowska ${ }^{1}$, \\ Wojciech Kucharski1,3, Jacek Stodółka², Krzysztof Maćkała²
}

\begin{abstract}
The aim of this study was to assess the efficacy of inspiratory muscle training (IMT) at different intensities on the pulmonary function and physiological adaptations of long-distance runners undergoing sports training. This study involved 25 long-distance runners. The subjects were randomly divided into three groups depending on the type of IMT applied: POWERbreathe device (group 1), Threshold IMT device (group 2), and a control group. The following lung variables were evaluated: vital capacity $(V C)$, forced vital capacity $(F V C)$, forced expiratory volume in one second $\left(F E V_{1}\right)$ and peak expiratory flow (PEF). Respiratory muscle strength was assessed by maximum inspiratory pressure (PImax) and maximum expiratory pressure (PEmax). Spiroergometric measures included: heart rate (HR), oxygen uptake $\left(V \mathrm{O}_{2 \max }\right)$, carbon dioxide production $\left(\mathrm{VCO}_{2 \max }\right)$, maximum ventilation (VE) and respiratory exchange rate (RER), which were measured breath by breath using a gas analyser (VYNTUS CPX). Group 1, which used the POWERbreathe device, showed significant increases in all assessed physiological and physical performance variables. In group 2, which used the Threshold device, only $V O_{2 m a x}, V E$ and $t R E R$ ventilation were significantly increased to a similar level as that observed in group 1. In the control group, we only observed a significant reduction in saturation. The use of IMT with a higher intensity resulted in significant improvements in all tested variables of lung ventilation and respiratory muscle strength. Also, after training, lactate accumulation was significantly decreased. Physiological characteristics $\left(V_{2} \mathrm{max}_{2} / \mathrm{kg}\right)$ and muscle respiratory strength variables were significantly improved in the group that used the POWERbreathe device after 8 weeks of training.
\end{abstract}

Key words: inspiratory muscle training; runners; pulmonary function; physiological adaptations.

\section{Introduction}

Athletics distance running training encompasses many aspects, all of which have a significant impact on the final outcome (Nepomuceno et al., 2016; Waśkiewicz et al., 2012). The training process itself is critical for optimising exercise performance, so as to achieve the optimum strength and power and prevent musculoskeletal injuries (Pastre et al., 2005). The role of the cardiovascular system is to maximise the perfusion capacity to prevent lactic acidosis, which reduces performance (Pastre et al., 2005; Plentz et al., 2012). However, respiratory muscle fatigue is frequently overlooked, and inspiratory muscle training (IMT) can be applied to help prevent fatigue (McConnell, 2009). The benefits of IMT are related to a greater supply of oxygen to the tissues, thereby enhanced physical performance (Moodie et al., 2011; Ribeiro et al., 2012). It is well known that respiratory muscles undergo fatigue during exercise, which limits their capacity and results in a poorer supply of oxygen to the working muscles. Therefore, it seems reasonable to investigate the function of respiratory muscles in professional athletes (Enright et al., 2006; McConnell, 2009; Wilson et

1 - Department of Physiotherapy of the University of Physical Education in Wroclaw, Poland.

2 - Department of Physical Education of the University of Physical Education in Wroclaw, Poland.

3 - Department of Internal Diseases EMC Medical Institute SA, Wroclaw, Poland. 
al., 2014). The respiratory system and its central regulation unit control the levels of oxygen $\left(\mathrm{O}_{2}\right)$ and carbon dioxide $\left(\mathrm{CO}_{2}\right)$, which are released at higher levels during power or endurance activities (Sheel et al., 2001; Tong et al., 2012; Turner et al., 2012). In a study on cyclists by Klusiewicz et al., (2008), it was observed that the diaphragm undergoes considerable fatigue during intensive work at a constant load $\left(80-85 \% \mathrm{VO}_{2 \max }\right)$ (Klusiewicz et al., 2008). This finding was confirmed by Voliantis et al. (2001) in rowers following a short, intensive training programme (Voliantis et al., 2001). Other authors have also confirmed that sports training should be complemented by respiratory muscle exercise (Jurić et al., 2019; Martins et al., 2019; McConnell and Sharpe, 2005).

Recent studies have suggested that the inspiratory muscles can affect athletes' physical performance. Under physically stressful conditions, when the production of free radicals exceeds the elimination capacity, the sympathetic peripheral vasoconstriction reflex is triggered, leading to early skeletal muscle fatigue and, consequently, a dramatic reduction in performance. Conditioned muscles, on the other hand, have an increased tolerance, which delays the activation of this reflex (McConnell et al., 2010; McConnell et al., 2006; Sheel et al., 2001).

Inspiratory muscle training is a therapeutic modality that overcomes resistance against the muscles responsible for expanding the rib cage. This effort must be controlled, specific and repeated at regular intervals. The application of IMT has been demonstrated to be a viable strategy for optimising respiratory capacity (Caine and McConnell, 2000).

Increasing evidence supports the use of IMT in athletes of various sports (Sheel et al., 2001; McConnell and Griffiths, 2010). The available literature often reports the use of IMT in sports like swimming, rowing and cycling, whereas only a few studies have reported its use in track and field athletes, particularly runners (Tong et al., 2012; HajGhanbari et al., 2013).

The use of IMT makes it possible to achieve better physiological functioning of the body, which can improve athletic results. There are no studies that clearly show whether IMT and the type of training equipment have an important impact on the preparation of high-level athletes.
There are many devices available for such training, however, no comparisons of the physiological effects obtained after their application have been performed.

The type of device used is important considering the possibility of increasing loads. In competitive sport, a POWERbreathe trainer should be used, whereas a Threshold device should be used for people with lower exercise capacity (e.g., patients in a rehabilitation programme).

Nevertheless, it is necessary to conduct systematic research based on consistent methodology to clarify the benefits of using IMT in sports training. In addition, knowledge about IMT, specifically its indication, dosage and benefits, are still not fully understood. Thus, the aim of this experiment was to assess the efficacy of IMT at different intensities (POWERbreathe and Threshold) on the pulmonary function and physiological adaptations of long-distance runners undergoing sports training. We hypothesised that IMT using the POWERbreathe device would promote greater improvements in performance and physiological adaptations compared to the Threshold device.

\section{Methods}

\section{Participants}

This study involved 25 athletes, all longdistance runners. The inclusion criteria for this study were: age between $18-28$ years, minimum 5 years of training experience, and elite sport level. The exclusion criteria were: presence of bronchial asthma or any other respiratory illness requiring the use of medication, history of spontaneous pneumothorax (collapsed lung due to an injury such as rib fracture), and a condition following the rupture of a tympanic membrane.

The subjects were randomly divided (distribution table) into three groups depending on the type of IMT applied. Group 1 consisted of 11 runners who received additional IMT using the POWERbreathe device, group 2 consisted of nine athletes who completed additional IMT using the Threshold IMT device, and group 3, the control group, consisted of five runners who performed their regular training. Detailed characteristics of the subjects are presented in Table 1. The three groups were homogeneous. No significant differences were found in the anthropometric 
variables between the assessed groups. All runners completed the same general preparatory training cycle.

Characteristics of long-distance runners

Success in long-distance running requires discipline, dedication and proper coaching/training. A well-designed plan can improve the quality of training and reduce the risk of injury as a result of running. The training carried out in this experiment was intended for advanced long-distance runners, including those who run $5 \mathrm{~km}, 10 \mathrm{~km}$ or even marathons. The training took place during general preparation $(8$ weeks) and special preparation (2 weeks) phases. During this period, the effective training plan (Table 2) assumes that the appropriate work is performed relating to general endurance, including the mileage covered in $65-79 \%$ of max heart rate [HR] or 1-2 mmol lactate [LA] below the anaerobic threshold. It also includes works for the mobility of the motor system, and appropriate strength levels. Late in this period the training goes into the quality of running in near the anaerobic threshold which is characterized by an effort of intensity range $75-85 \%$ of max HR or $2-4$ mmol LA. The final stage of training in this period is representing high-quality workouts, where running is above the anaerobic threshold, range $85-95 \%$ of max HR or 8-10 mmol LA. These activities moreover must also account the right number of recovery days.

\section{Measurements}

Each competitor underwent two tests, the first was conducted immediately before starting the general preparation phase and the second after the end of the cycle (i.e., after 2 months). All athletes had their height and body weight measurements recorded, and functional examination of their respiratory system, respiratory muscle strength (based on the measurement of PImax and PEmax values) and physical capacity (spiroergometric test) was also performed.

\section{Design and procedures}

Evaluation of the subjects' respiratory function was carried out using the MasterScreen Pneumo apparatus (CareFusion, Germany). A forced expiratory technique was performed, during which the flow/volume curve was recorded. The tests were performed in accordance with the American Thoracic Society and the
European Respiratory Society (ATS/ERS) criteria (ATS/ERS, 2002). The forced expiratory test was performed three times. The exhalation time lasted a minimum of $6 \mathrm{~s}$, and at least two of the three breathing manoeuvres had to be repeatable, that is, the forced vital capacity (FVC) and forced expiratory volume in one second $\left(\mathrm{FEV}_{1}\right)$ did not differ by more than $100 \mathrm{ml}$ or $5 \%$, respectively. The absolute values measured during the study referred to normalised values (Quanjer et al., 1993), that is the values of individual lung function variables calculated hypothetically based on morphometric parameters such as sex, age, height and, to a lesser extent, body weight. The regression formula inputted into the Jaeger Electronic Masterscreen Spirometer to calculate the normal values correspond to the standards developed by the European Community for Coal and Steel, Luxemburg (Degens and Merget, 2008).

The following variables were selected for analysis: vital capacity (VC) [1], forced vital capacity (FVC) [1], forced expiratory volume in one second $\left(\mathrm{FEV}_{1}\right)$ and peak expiratory flow (PEF) [1/s].

Assessment of respiratory muscle strength was performed by measuring the maximum inspiratory pressure (PImax or $\mathrm{MIP} ; \mathrm{kPa}$ ) and maximum expiratory pressure (PEmax or MEP; $\mathrm{kPa})$, which represent the maximum pressure values during the first second of inhalation and exhalation, respectively. In order to correctly measure the PImax and PEmax values, a special MasterScreen Pneumo adapter was used. The test was carried out in accordance with the 2002 ATS/ERS guidelines. The subjects were instructed to perform a maximum inhalation for 2-3 s. The test was carried out in a sitting position. In accordance with standard procedure, 5 to 10 correct measurements were performed and the three highest values with less than $5 \%$ variation were selected. The strength of the inspiratory muscles measured by the apparatus was expressed in kilopascals $(\mathrm{kPa})$. For the inspiratory training, it was necessary to convert the values into centimetres of water $\left(\mathrm{cm} \mathrm{H}_{2} \mathrm{O}\right)$ according to the formula $1 \mathrm{kPa}=10.2 \mathrm{~cm} \mathrm{H}_{2} \mathrm{O}$ (ATS/ERS, 2002).

Assessment of the athletes' physical capacity was carried out by conducting a maximum progressive test on a treadmill. Subjects were familiar with this protocol as it is commonly used for physical evaluations during the season. 
The protocol was carried out on a treadmill with a fixed inclination of $1 \%$. The protocol started with a $5 \mathrm{~min}$ warm-up at $6 \mathrm{~km} / \mathrm{h}$. For the main part of the assessment, the belt velocity was increased by $2 \mathrm{~km} / \mathrm{h}$ every $3 \mathrm{~min}$. After reaching $16 \mathrm{~km} / \mathrm{h}$, the velocity was kept constant, but the incline of the treadmill was increased by $2.5 \%$ every $3 \mathrm{~min}$. The protocol is volitional, and competitors were provided verbal encouragement to reach maximum exercise intensity. The criteria for terminating the test included voluntary exhaustion or reaching the subject's maximum HR (calculated as $220 \mathrm{bpm}$ - age). The oxygen uptake $\left(\mathrm{VO}_{2 \max }\right)$, carbon dioxide production $\left(\mathrm{VCO}_{2 \max }\right)$, maximum ventilation (VE) and respiratory exchange rate (RER) were measured breath by breath using a gas analyser (VYNTUS (PX). Heart rate was recorded using a transmission tape with a sensor and interface (Polar ${ }^{\circledR}$, Finland). A known gas mixture $\left(12 \% \mathrm{O}_{2}+\right.$ $5 \% \mathrm{CO}_{2}$ ) was used to calibrate the gas analyser before each test (ATS/ERS, 2002). Capillary blood samples $(0.2 \mu \mathrm{l})$ were taken from the fingertips of the athletes. The lactate threshold (LT) was analysed immediately using a portable analyser (Lactate Scount+, Germany).

Inspiratory muscle training using the POWERbreathe device

Athletes in group 1 underwent IMT for 8 weeks, with two sessions performed daily, five times a week (preceding specific team training). To perform IMT, the group carried out 30 maximum inspirations, maintaining the diaphragmatic inspiratory muscle pattern (Sheel et al., 2001). One session per week was performed under supervision, and the other four sessions were performed at home. A threshold loading device (POWERbreathe KH1; POWERbreathe International Ltd., England) was used to ensure inspiratory muscle loading.

For group 1, the IMT program was carried out using the following protocol: from week 1 to 3 an intensity of $50 \%$ of the MIP was used, from week 4 to 6 the intensity was $60 \%$ of the MIP, and from week 7 to 8 the intensity was increased to $70 \%$ of the MIP (ATS/ERS, 2002).

Inspiratory muscle training using the Threshold device

Athletes in group 2 performed IMT using the Threshold device for 8 weeks, with two sessions daily, five times a week (preceding specific team training). The group performed 30 maximum inspirations, maintaining the diaphragmatic inspiratory muscle pattern (Janssens et al., 2013). For group 2, the IMT programme started with a load of $30 \%$ of the PImax, which was increased weekly up to $50 \%$ of the PImax (Agnihotri et al., 2016).

\section{Statistical analysis}

The results were analysed using Statistica version 7.0 software by StatSoft (Dell, Round Rock, TX, USA). The Kolmogorov-Smirnov test and the Lilliefors test were used to assess the normality of distribution. Basic descriptive statistics were calculated.

Differences in respiratory system and physical capacity variables between the groups and the tests were evaluated using repeatedmeasures ANOVA and NIR post hoc test. Differences at $p<0.05$ were considered statistically significant.

\section{Results}

There was_a significant increase in most of the lung ventilation variables themfollowing IMT using the POWERbreathe device (Tables 3 and 4). In group 2, in which the Threshold device was used, only the vital capacity of lungs increased significantly. In the control group, in which only typical sports training was performed, none of the variables changed significantly.

In Group 1, following additional training with the POWERbreathe device (Tables 5) led to significant increases in all physiological and physical performance variables. In group 2 , which received additional training with the Threshold device, only the $\mathrm{VO}_{2 \max }$ (expressed in millilitres per minute) and VE1 and tRER (expressed in minutes) ventilation were significantly increased to a similar level as that observed in group 1. In the control group, which performed typical sports training, we only observed a significant reduction in saturation.

The post-hoc tests to assess the presence of significant differences between the groups revealed a significantly higher value for FVC in athletes who trained with the POWERbreathe training device compared to the control group $(p=$ 0.001 ), and FVC also tended to be higher than the Threshold training group $(p=0.062)$.

The value of maximal inspiratory pressure (PImax) after the POWERbreathe training was significantly higher than in the group that 
performed Threshold training $(p=0.001)$ and the control group $(p=0.002)$.

The value of maximal expiratory pressure (PEmax) after POWERbreathe training was significantly higher than in the Threshold training group $(p=0.002)$ and the control group $(p=0.007)$.

The value of oxygen uptake per kilogram $\left(\mathrm{VO}_{2} / \mathrm{kg}\right)$ was significantly higher after training with the POWERbreathe device than after Threshold training $(p=0.011)$, and was also higher than the control group $(p=0.014)$.

The HR value in the group that performed POWERbreathe training was significantly lower than in the Threshold training $(p=0.001)$ and control $(p=0.01)$ groups.

The oxygen saturation level $\left(\mathrm{SpO}_{2}\right)$ value of athletes following POWERbreathe training was significantly higher than those for Threshold training $(p=0.002)$ and the control group ( $p=$ 0.003).

The value for lactate threshold after the POWERbreathe training was significantly higher than the Threshold training group $(p=0.007)$ and the control group $(p=0.043)$.

The value of maximum ventilation $\left(\mathrm{VE}_{1}\right)$ after POWERbreathe training was significantly higher than in the control group ( $p=0.023)$. However, the VE1 was significantly lower after Threshold training than in the control group ( $p=$ $0.021)$, and there was no difference between the POWERbreathe and Threshold groups $(p=0.881)$.

\section{Table 1}

Characteristics of the athletes in each group.

\begin{tabular}{lccc}
\hline Variable & $\begin{array}{c}\text { POWERbreathe } \\
(\mathrm{n}=11)\end{array}$ & $\begin{array}{c}\text { Threshold } \\
(\mathrm{n}=9)\end{array}$ & $\begin{array}{c}\text { Control } \\
(\mathrm{n}=5)\end{array}$ \\
\hline Sex & $6 \mathrm{~F} / 5 \mathrm{M}$ & $4 \mathrm{~F} / 5 \mathrm{M}$ & $3 \mathrm{~F} / 2 \mathrm{M}$ \\
Training (yrs) & 7.25 & 5.5 & 6.25 \\
Age (yrs) & $23.73 \pm 2.1$ & $23.89 \pm 3.26$ & $23.20 \pm 1.92$ \\
Height (m) & $1.76 \pm 0.07$ & $1.76 \pm 0.08$ & $1.75 \pm 0.08$ \\
Body mass (kg) & $65.27 \pm 7.24$ & $69.44 \pm 7.65$ & $67.80 \pm 8.35$ \\
BMI (kg/m $\mathbf{2})$ & $20.94 \pm 1.00$ & $22.34 \pm 1.33$ & $21.95 \pm 0.72$ \\
\hline
\end{tabular}

Abbreviations: Mean $\pm S D ; B M I-b o d y$ mass index

Table 2

Long-distance training programme in each weekly microcycle during the preparation phase.

Type of training modality (workout)

Training session (7-day microcyle)

Long distance

Number of performance $(n)$ Mileage (km)

Strength Endurance (combined with circuit training)

GO1 (general endurance: $65-79 \%$ max HR or 1-2 mmol LA)

$60-70$

GO2 (general durance: $75-85 \%$ max HR or $2-4$ mmol LA)

SP (special endurance: $85-95 \%$ max HR or 6-8 mmol LA)

Tempo (interval training: $85-95 \%$ max HR or $8-10 \mathrm{mmol}$

LA)

SRE (strength running endurance: skips and hill running)

General fitness (supplementary session)

Recovery (swimming, massage, cryotherapy)

Day off (rest)

Total training workouts per module

10-15

5-10

$5-10$

2-3

$-$

$82-108$

Abbreviations: LA-lactate 
Table 3

Mean and standard deviation (SD) of lung function variables in the studied groups.

Results of the LSD post-hoc analysis of the function between tests 1 and 2 for groups that trained with the POWERbreathe device, the Threshold device and the control group.

\begin{tabular}{|c|c|c|c|c|c|}
\hline \multicolumn{2}{|c|}{ POWERbreathe } & \multicolumn{2}{|c|}{ Threshold } & \multicolumn{2}{|c|}{ Control } \\
\hline Pre-training & Post-training & Pre-training & Post-training & Pre-training & Post-training \\
\hline \multicolumn{6}{|c|}{ VC (1) } \\
\hline $4.02 \pm 0.78$ & $4.30 \pm 0.94$ & $3.50 \pm 0.54$ & $3.77 \pm 0.63$ & $3.52 \pm 0.89$ & $3.7 \pm 0.65$ \\
\hline \multicolumn{2}{|c|}{$.023^{*}$} & \multicolumn{2}{|c|}{$.043^{*}$} & \multicolumn{2}{|c|}{.183} \\
\hline \multicolumn{6}{|c|}{ FVC (1) } \\
\hline $4.48 \pm 0.56$ & $4.63 \pm 0.59$ & $4.18 \pm 0.61$ & $4.12 \pm 0.59$ & $3.71 \pm 0.48$ & $3.55 \pm 0.49$ \\
\hline \multicolumn{2}{|c|}{.085} & \multicolumn{2}{|c|}{.506} & \multicolumn{2}{|c|}{.200} \\
\hline \multicolumn{6}{|c|}{$\mathrm{FEV}_{1}(\mathbf{1} / \mathrm{s})$} \\
\hline $4.05 \pm 0.51$ & $4.36 \pm 0.56$ & $4.02 \pm 0.44$ & $4.07 \pm 0.59$ & $4.25 \pm 0.58$ & $4.28 \pm 0.64$ \\
\hline \multicolumn{2}{|c|}{$.000^{*}$} & \multicolumn{2}{|c|}{.530} & \multicolumn{2}{|c|}{.818} \\
\hline \multicolumn{6}{|c|}{$\operatorname{PEF}(1 / s)$} \\
\hline $6.42 \pm 1.13$ & $6.61 \pm 1.09$ & $6.68 \pm 0.96$ & $6.67 \pm 0.89$ & $6.15 \pm 1.30$ & $6.10 \pm 1.26$ \\
\hline \multicolumn{2}{|c|}{$.011^{*}$} & \multicolumn{2}{|c|}{.914} & \multicolumn{2}{|c|}{.625} \\
\hline
\end{tabular}

Abbreviations: $V C$, vital capacity; $F V C$, forced vital capacity; $F E V_{1}$, forced expiratory volume in one second; PEF, peak expiratory flow.

Values are expressed as mean $\pm S D$. ${ }^{*}$ Significant difference.

Table 4

Mean and standard deviation (SD) of respiratory muscle strength variables in the studied groups. Results of the LSD post-hoc analysis of the respiratory muscles between tests 1 and 2 for groups that trained with the POWERbreathe device, the Threshold device and the control group.

\begin{tabular}{|c|c|c|c|c|c|}
\hline \multicolumn{2}{|c|}{ POWERbreathe } & \multicolumn{2}{|c|}{ Threshold } & \multicolumn{2}{|c|}{ Control } \\
\hline Pre-training & Post-training & Pre-training & Post-training & Pre-training & Post-training \\
\hline \multicolumn{6}{|c|}{$P I_{\max }(\mathbf{k P a})$} \\
\hline $9.77 \pm 1.82$ & $12.32 \pm 2.16$ & $9.49 \pm 2.31$ & $9.01 \pm 1.80$ & $8.94 \pm 1.54$ & $8.62 \pm 1.50$ \\
\hline \multicolumn{2}{|c|}{$.000^{*}$} & \multicolumn{2}{|c|}{.093} & \multicolumn{2}{|c|}{.396} \\
\hline \multicolumn{6}{|c|}{$\mathrm{PE}_{\max }(\mathbf{k P a})$} \\
\hline $9.57 \pm 2.07$ & $12.14 \pm 2.81$ & $9.20 \pm 1.49$ & $8.97 \pm 1.75$ & $8.90 \pm 1.77$ & $8.82 \pm 1.76$ \\
\hline \multicolumn{2}{|c|}{$.000^{*}$} & \multicolumn{2}{|c|}{.556} & \multicolumn{2}{|c|}{.876} \\
\hline
\end{tabular}

Abbreviations: PI $I_{\max }$ inspiratory muscle strength; PEmax - expiratory muscle strength. Values are expressed as mean $\pm S D .{ }^{*}$ Significant difference. 
Table 5

Mean and standard deviation (SD) of physiological parameters in the studied groups. Results of the LSD post-hoc analysis of the between tests 1 and 2 for groups that trained with the POWERbreathe device, the Threshold device and the control group.

\begin{tabular}{|c|c|c|c|c|c|}
\hline \multicolumn{2}{|c|}{ POWERbreathe } & \multicolumn{2}{|c|}{ Threshold } & \multicolumn{2}{|c|}{ Control } \\
\hline Pre-training & Post-training & Pre-training & Post-training & Pre-training & Post-training \\
\hline \multicolumn{6}{|c|}{$\mathrm{VO}_{2 \max }(\mathrm{ml} / \mathrm{min})$} \\
\hline $\begin{array}{c}3723.55 \pm \\
804.23\end{array}$ & $\begin{array}{c}4047.64 \pm \\
793.07\end{array}$ & $\begin{array}{c}3613.56 \pm \\
753.56\end{array}$ & $\begin{array}{c}3734.89 \pm \\
750.81\end{array}$ & $\begin{array}{c}3402.20 \pm \\
814.38\end{array}$ & $\begin{array}{c}3387.80 \pm \\
832.34\end{array}$ \\
\hline \multicolumn{2}{|c|}{$.000^{*}$} & \multicolumn{2}{|c|}{$.001^{*}$} & \multicolumn{2}{|c|}{.749} \\
\hline \multicolumn{6}{|c|}{$\mathrm{VO}_{2} / \mathrm{kg}[(\mathrm{ml} / \mathrm{min}) / \mathrm{kg}]$} \\
\hline $56.07 \pm 6.75$ & $60.86 \pm 6.43$ & $53.02 \pm 3.26$ & $54.06 \pm 2.98$ & $53.24 \pm 2.99$ & $53.04 \pm 3.26$ \\
\hline \multicolumn{2}{|c|}{$.000^{*}$} & \multicolumn{2}{|c|}{.358} & \multicolumn{2}{|c|}{.894} \\
\hline \multicolumn{6}{|c|}{$\mathrm{VCO}_{2 \max }(\mathrm{ml} / \mathrm{min})$} \\
\hline $\begin{array}{c}4427.09 \pm \\
1121.89\end{array}$ & $\begin{array}{c}4309.27 \pm \\
1072.47\end{array}$ & $\begin{array}{c}4350.56 \pm \\
1288.90\end{array}$ & $\begin{array}{c}4278.78 \pm \\
1423.69\end{array}$ & $\begin{array}{c}3465.00 \pm \\
1194.93\end{array}$ & $\begin{array}{c}3578.00 \pm \\
1216.97\end{array}$ \\
\hline \multicolumn{2}{|c|}{.208} & \multicolumn{2}{|c|}{.572} & \multicolumn{2}{|c|}{.630} \\
\hline \multicolumn{6}{|c|}{ HRmax (bpm) } \\
\hline $188.36 \pm 7.31$ & $181.64 \pm 6.87$ & $191.44 \pm 6.52$ & $191.56 \pm 6.43$ & $191.40 \pm 5.86$ & $190.60 \pm 5.13$ \\
\hline \multicolumn{2}{|c|}{$.000^{*}$} & \multicolumn{2}{|c|}{.064} & \multicolumn{2}{|c|}{.701} \\
\hline \multicolumn{6}{|c|}{ Lactate (mmol) } \\
\hline $8.61 \pm 2.32$ & $7.26 \pm 1.71$ & $8.43 \pm 1.49$ & $8.29 \pm 1.34$ & $7.91 \pm 1.53$ & $8.28 \pm 1.69$ \\
\hline \multicolumn{2}{|c|}{$.000^{*}$} & \multicolumn{2}{|c|}{.566} & \multicolumn{2}{|c|}{.268} \\
\hline \multicolumn{6}{|c|}{$\mathrm{SpO}_{2}(\%)$} \\
\hline $96.00 \pm 0.89$ & $97.64 \pm 0.81$ & $95.89 \pm 1.36$ & $96.00 \pm 1.58$ & $97.20 \pm 0.84$ & $95.80 \pm 1.10$ \\
\hline \multicolumn{2}{|c|}{$.001^{*}$} & \multicolumn{2}{|c|}{.823} & \multicolumn{2}{|c|}{$.041^{*}$} \\
\hline \multicolumn{6}{|c|}{ LT (min) } \\
\hline $12.82 \pm 1.08$ & $17.09 \pm 3.98$ & $13.45 \pm 0.77$ & $13.53 \pm 1.39$ & $13.40 \pm 1.68$ & $12.90 \pm 3.15$ \\
\hline \multicolumn{2}{|c|}{$.000^{*}$} & \multicolumn{2}{|c|}{.928} & & \\
\hline & & Lo & (W) & & \\
\hline $243.18 \pm 82.48$ & $263.73 \pm 88.18$ & $288.33 \pm 98.81$ & $327.56 \pm 107.53$ & $240.40 \pm 93.84$ & $242.60 \pm 93.94$ \\
\hline & & & & & \\
\hline & & $\mathrm{VE}_{1}$ & $\min )$ & & \\
\hline $\begin{array}{c}102.82 \pm \\
17.25\end{array}$ & $122.73 \pm 24.60$ & $110.78 \pm 22.58$ & $124.22 \pm 26.55$ & $93.60 \pm 12.54$ & $93.80 \pm 12.32$ \\
\hline & & & & & \\
\hline & & t RE & $(\min )$ & & \\
\hline $13.54 \pm 5.70$ & $17.83 \pm 4.95$ & $14.15 \pm 15.03$ & $17.14 \pm 18.45$ & $18.74 \pm 5.76$ & $19.35 \pm 5.32$ \\
\hline & & & & & \\
\hline
\end{tabular}

Abbreviations: $\mathrm{VO}_{2}$ max, maximal oxygen consumption; $\mathrm{VO}_{2} / \mathrm{kg}$, oxygen uptake per $\mathrm{kg}$;

$\mathrm{VCO}_{2}$ max, carbon dioxide production; $\mathrm{HR}$, heart rate; $\mathrm{SpO}_{2}$, oxygen saturation levels; $L T$, lactate threshold; $V E_{1}$, maximum ventilation; $t R E R$, time of respiratory exchange ratio. Values are expressed as mean $\pm S D$. ${ }^{*}$ Significant difference. 


\section{Discussion}

The application of IMT using the POWERbreathe device improved respiratory function, evidenced by a significant increase in all lung ventilation variables in this group of runners. So far, there has been no consensus in the literature regarding the positive effects of IMT on resting lung function volume. One of the main findings of the current study was the confirmation of a significant impact of an intensive 8-week IMT program, which resulted in increased resting pulmonary volumes in this group of subjects.

HajGhanbari et al. (2013) performed a systematic review and meta-analysis of 21 randomised controlled trials on the effect of various types of inspiratory training on lung function, but only a positive effect on $\operatorname{FEV}_{1}(p=$ 0.02) was demonstrated. The lung function results can be explained by a decrease in upper airway resistance and an increase in diaphragm strength as a result of training (HajGhanbari et al., 2013).

Consistent with previous studies, it is possible that the addition of IMT to physical training might have led to further improvements in the physical performance of competitors in group 1 (POWERbreathe) compared to those who trained with the Threshold device and to the control group. POWERbreathe ${ }^{\circledR}$ is one of many available devices on the market that generate resistance through a spring system or electronic valve.

The main difference between this device and others is the ability to set the largest load during training and adjust the inspiratory resistance to the pulmonary pressure curve, which can generate load stabilisation during breathing, making it more comfortable for the subject (McConnell and Sharpe, 2005). On the other hand, Threshold is a threshold-loading device designed for frail patients or those with chronic lung disorders. These devices offer a maximum training resistance of approximately 41 $\mathrm{cm} \mathrm{H}_{2} \mathrm{O}$. At a training intensity of $50 \% \mathrm{PI}_{\max }$, the resistance of $41 \mathrm{~cm} \mathrm{H}_{2} \mathrm{O}$ offered by these threshold devices is clearly insufficient to provide adequate loading to improve the inspiratory muscle strength of healthy individuals, such as the athletes in this study (Kwok and Jones, 2009).

Inspiratory muscle training program that are regularly repeated and include a controlled and individualised load result in the growth of sarcomeres while also increasing muscle volume and strength, which results from muscle physiology (Nepomuceno et al., 2016). In line with this, IMT training in the current study resulted in improvements in PImax in comparison to the group that trained with the Threshold device, which improved to a lesser extent, and to the control group, with an increase of $10 \%$ observed after 8 weeks of training.

The use of IMT devices has been evaluated as an addition to sports training in various disciplines, including swimming, rowing, cycling, soccer, running and tennis (Caine and McConnell, 2000; Griffiths and McConnell, 2007; Romer et al., 2002; Volianitis et al., 2001). In research on athletes who used POWERbreathe as additional IMT, McConnell et al. (2010) and Romer et al. (2002) observed a decrease in HR, respiratory rate and feelings of dyspnoea, according to Borg's rating of perceived exertion (RPE) (McConnell and Griffiths, 2010; Romer et al., 2002). These results suggest that the use of IMT can improve exercise tolerance during sports training. In addition, Romer et al. (2002) showed an improvement in the $\mathrm{VO}_{2 \max }$ of cyclists after 6 weeks of training compared to a placebo.

The results obtained in the current study support a significant improvement in the physical performance of athletes when high-intensity IMT is added to their training programme. However, the application of inspiratory muscle resistance training at a lower intensity (group 2) does not affect physical performance.

Sheel et al. (2001) showed that inspiratory muscle fatigue activates the reactive sympathetic peripheral vasoconstriction response, which could accelerate muscle fatigue in the extremities during physical activity (Sheel et al., 2001), a condition known as metaboreflex. In a clinical trial using various IMT protocols, McConnell and Lomax (2006) observed early fatigue of the lower extremities in groups undergoing high-load limb training (general preparation phase of an athlete), which resulted in significant overload of the respiratory muscles (McConnell and Lomax, 2006). This is why the addition of IMT to a normal training program seems valuable, as it can reduce the incidence of muscle fatigue and improve the performance of the lower extremities, which is particularly important for runners.

All of the studies cited above tested 
interventional protocols including 30 breaths with inhalation resistance, equivalent to $56 \%$ MIP, repeated two times per day, seven days a week. An equivalent resistance was applied in the current study, the only difference was that it was applied for 5 days instead of 7 days per week, making it possible for comparison between studies.

Based on their studies, Harms et al. (1997) and McConnell et al. (2006) proposed that the improvement in exercise capacity following IMT may be the result of improved blood flow to the active locomotor muscles, which could delay the onset of peripheral muscle fatigue. Following IMT, the improvement in blood flow to the skeletal muscles delays the build-up of lactate and fatigue in the active muscles. As a result, competitors could complete a trial while working at a faster speed for a longer time (Harms et al., 1997; McConnel et al., 2006).

The results of this research also confirmed a reduction in lactate production in competitors who trained using the POWERbreathe device, confirming the possibility of delayed skeletal muscle fatigue and resulting in a reduction in exercise HR in this group. Together with the aforementioned studies, this research confirms that IMT can change breathing mechanics, leading to improved lung ventilation and alleviating the oxygen cost of physical exertion, as well as lowering blood lactate levels (Harms et al., 1997; McConnell and Lomax, 2006).

\section{Perspectives}

The addition of inspiratory muscle training to the typical training programme for long-distance runners can enhance aerobic capacity. We propose that higher intensity training employing the POWERbreathe device contributes to better athletic performance by improving all lung ventilation variables, respiratory muscle strength and performance parameters, and by reducing lactate levels. It is also important to properly adapt the IMT program to the sport training cycle for runners. We propose that IMT be applied during the general preparatory cycle due to the benefits obtained.

\section{Acknowledgements}

We would like to thank the athletes for the participation in this study and the coach: Jacek Wosiek for his cooperation. This trial was funded by University Sport Development Grant ID: 288418. Registration number: N RSA4 05454

\section{Practical implications}

The addition of IMT into a typical training cycle for runners constitutes an important rounding-off to the sports training programme. To date, only a few reports have been carried out on runners, and their conclusions have not entirely confirmed an improvement in lung ventilation parameters. Moreover, the type of IMT and the resistance used for this training were not uniform in terms of the achieved effects. The current study confirms that use of the POWERbreathe device with a resistance of 50$70 \%$ of MIP provided the greatest benefits, significantly increasing the values of lung ventilation variables $\left(\mathrm{VC}, \mathrm{FVC}, \mathrm{FEV}_{1}, \mathrm{PEF}\right.$ and $\mathrm{MEF}_{50}$ ), respiratory muscle strength (PImax and PEmax), physical performance $\left(\mathrm{VO}_{2 \max }, \mathrm{VO}_{2} / \mathrm{kg}\right.$, lactate threshold, load, VE and t RER) and the body's response to physical exercise (lactate, $\mathrm{SpO}_{2}$, and HR). Based on the above results, inspiratory muscle training using POWERbreathe in long-distance runners may be significant in achieving better sport results.

\section{Limitations}

The biggest limitation of this study was the small number of runners in particular groups, which was limited by the total number of players with an appropriate sports level at a single sports club.

\section{Conclusion}

1. The use of IMT with a higher intensity resulted in significant improvements in all tested variables of lung ventilation and respiratory muscle strength.

2. Physiological characteristics $\left(\mathrm{VO}_{2 \max } / \mathrm{kg}\right)$ and muscle respiratory strength parameters were significantly increased after 8 weeks of training in the group that used the POWERbreathe device.

3. Plasma lactate accumulation was significantly decreased, which may indicate enhanced performance of athletes. 


\section{References}

Agnihotri DS, Bhise AR, Patel SM. Effect of Inspiratory Muscle Trainer on Running Performance and Respiratory Muscle Strength in Athletes. IAIM, 2016; 3(8): 159- 163

American Thoracic Society/European Respiratory Society ATS/ERS Statement on respiratory muscle testing. Am J Respir Crit Care Med, 2002; 166: 518-624

Bissett B, Leditschke IA. Anaesth Intensive Care. Inspiratory muscle training to enhance weaning from mechanical ventilation. 2007 Oct; 35 (5): 776 - 779

Caine MP, McConnell AK. Development and evalution of a pressure threshold inspiratory muscle trainer for use in the context of sports performance. Sports Eng, 2000; 3: 149 - 159

Degens P, Merget R. Reference values for spirometry of the European Coal and Steel Community: time for change. Eur Respir J. 2008 Mar; 31 (3): 687 - 688; author reply 688-689

Edwards AM. Respiratory muscle training extends exercise tolerance without concomitant change to peak oxygen uptake: physiological, performance and perceptual responses derived from the same incremental exercise test. Respirology 2013, 18, $1022-1027$

Enright SJ, Unnithan V, Heward C, Withnall L, Davies DH. Effect of high-intensity inspiratory muscle training on lung volumes, diaphragm thickness, and exercise capacity in subjects who are healthy. Phys Ther 2006; 86: 345 -354

Griffiths LA, McConnell AK. The influence of inspiratory and expiratory muscle training upon rowing performance. Eur J Appl Physiol, 2007; 99(5): 457 - 466

HajGhanbari B, Yamabayashi C, Buna TR, Coelho JD, Freedman KD, Morton TA, Palmer SA, Toy MA, Walsh C, Sheel AW, Reid WD. Effects of Respiratory Muscle Training on Performance in Athletes: A Systematic Review With Meta-Analyses. J Strength Cond Res, 2013; 27 (6): 1643 - 1663

Harms CA, Babock MA, McClaran SR. Respiratory muscle work compromises leg blood flow during maximal exercise. J Appl Physiol, 1997; 82: 1573 - 1583

Janssens L, Brumagne S, Mcconnell AK, Raymaekers J, Goossens N, Gayan-Ramirez G, Hemans G, Trooters T. The assessment of inspiratory muscle fatigue in healthy individuals: A systematic review. Respir Med, 2013; 107: 331 - 346

Jurić I, Labor S, Plavec D, Labor M. Inspiratory muscle strength affects anaerobic endurance in professional athletes. Arh Hig Rada Toksikol, 2019 1; 70 (1): 42-48

Klusiewicz A, Borkowski L, Zdanowicz R, Boros P, Wesołowski S. The inspiratory muscle training in elite rowers. J Sports Med Phys Fitness, 2008; 48 (3): 279 - 284

Kwok T, Jones A. Target-flow inspiratory muscle training improves running performance in recreational runners: a randomized controlled trial. Hong Kong Physiother J, 2009; 27: 48 - 54

Martins de Abreu R, Porta A, Rehder-Santos P, Cairo B, Donisete da Silva C, De Favari Signini É, Sakaguchi CA, Catai AM. Effects of inspiratory muscle-training intensity on cardiovascular control in amateur cyclists. Am J Physiol Regul Integr Comp Physiol, 2019 1; 317(6): 891-902

McConnell AK, Griffiths LA. Acute Cardiorespiratory Responses to Inspiratory Pressure Threshold Loading. Med Sci Sports Exerc, 2010; 42 (9): 1696 - 1703

McConnell AK, Lomax M. The influence of inspiratory muscle work history and specific inspiratory muscle training upon human limb muscle fatigue. J Physiol, 2006; 577: 445 - 457

McConnell AK, Sharpe GR. The effect of inspiratory muscle training upon maximum lactate steady-state and blood lactate concentration. Eur J Appli Physiol, 2005; 94: 277 - 284

McConnell AK. Respiratory muscle training as an ergogenic aid. J Exerc Sci Fit, 2009; 7(2): 18 - 27

Moodie LH, Reeve JC, Vermeulen N, Elkins MR. Inspiratory muscle training to facilitate weaning from mechanical ventilation: protocol for a systematic review. BMC Res Notes, 2011; 4: 283

Nepomuceno Jr BRV, Gómez TB, Gomes Neto M. Use of Powerbreathe ${ }^{\circledR}$ in inspiratory muscle training for athletes: systematic review. Fisioter Mov, 2016; 29 (4): 821-830

Pastre CM, Carvalho Filho G, Monteiro HL, Netto Jr J, Padovani CR. Sports injuries in Brazilian elite of the athletics: study based on referred morbidity. Rev Bras Med Esporte, 2005; 11(1): 43-47

Plentz RD, Sbruzzi G, Ribeiro RA, Ferreira JB, Lago PD. Inspiratory Muscle Training in Patients with Heart Failure: Meta-Analysis of Randomized Trials. Arq Bras Cardiol, 2012; 99(2): 762-771 
Quanjer PH, Tammeling GJ, Cotes JE, Pedersen OF, Peslin R, Yernault JC Lung volumes and forced ventilatory flows. Eur Respir J, 1993; 6 (16): 5-40

Ribeiro JP, Chiappa GR, Callegaro CC. The contribution of inspiratory muscles function to exercise limitation in heart failure: pathophysiological mechanisms. Rev Bras Fisioter, 2012; 16(4): 17-25

Romer LM, McConnell AK, Jones DA Effects of inspiratory muscle training on time-trial performance in trained cyclists. J Sports Sci, 2002; 20: 547-562

Sheel AW, Derchak PA, Morgan BJ, Pegelow DF, Jacques AJ, Dempsey JA. Fatiguing inspiratory muscle work causes reblex reduction in resting leg blood flow in humans. J Physiol, 2001; 537: 277-289

Tong TK, Lin H, McConnell A, Eston R, Zheng J, Nie J. Respiratory and locomotor muscle blood-volume and oxygenation kinetics during intense intermittent exercise. Eur J Sport Sci, 2012; 12(4): 321-330

Turner LA, Tecklenburg-Lund SL, Chapman RF, Stager JM, Wilhite DP, Mickleborough TD. Inspiratory muscle training lowers the oxygen cost of voluntary hyperpnea. J Appl Physiol, 2012; 112(1): 127-134

Volianitis S, McConnell AK, Koutedakis Y, McNaughton L, Backx K, Jones DA. Inspiratory muscle training improves rowing performance. Med Sci Sports Exerc, 2001; 33(5) : 803-809

Waśkiewicz Z, Kłapcińska B, Sadowska-Krępa E, Czuba M, Kempa K, Kimsa E, Gerasimuk D. Acute metabolic responses to a 24-h ultra-marathon race in male amateur runners. Eur J Appl Physiol, 2012; 112 (5) : 1679-1688

Wilson E, McKeever TM, Lobb C, Sherriff T, Gupta L, Hearson G, Martin N, Lindley M.R, Shaw DE. Respiratory muscle specific warm-up and elite swimming performance. Br J Sports Med, 2014; 48(9): 789-791

\section{Corresponding author:}

\section{Paulina Okrzymowska, MSc}

Department of Physiotherapy in Internal Diseases

University School of Physical Education in Wroclaw

al.I.J.Paderewskiego 35

51-612 Wrocław, Poland

Tel. +48696597975

E-mail: paulina.okrzymowska@awf.wroc.pl 